Article

\title{
In Silico Analysis of Microarray-Based Gene Expression Profiles Predicts Tumor Cell Response to Withanolides
}

\author{
Thomas Efferth $^{1, *}$ and Henry Johannes Greten ${ }^{2,3}$
}

1 Department of Pharmaceutical Biology, Institute of Pharmacy and Biochemistry, Johannes Gutenberg University, Staudinger Weg 5, Mainz 55128, Germany

2 Heidelberg School of Chinese Medicine, Karlsruher Straße 12, Heidelberg 69126, Germany; E-Mail: heidelbergschool@aol.com

3 Biomedical Sciences Institute Abel Salazar, University of Porto, Porto 4050-313, Portugal

* Author to whom correspondence should be addressed; E-Mail: efferth@uni-mainz.de; Tel.: +49-6131-392-5751; Fax: +49-6131-392-3752.

Received: 19 April 2012; in revised form: 9 May 2012 / Accepted: 15 May 2012 /

Published: 22 May 2012

Abstract: Withania somnifera (L.) Dunal (Indian ginseng, winter cherry, Solanaceae) is widely used in traditional medicine. Roots are either chewed or used to prepare beverages (aqueous decocts). The major secondary metabolites of Withania somnifera are the withanolides, which are C-28-steroidal lactone triterpenoids. Withania somnifera extracts exert chemopreventive and anticancer activities in vitro and in vivo. The aims of the present in silico study were, firstly, to investigate whether tumor cells develop cross-resistance between standard anticancer drugs and withanolides and, secondly, to elucidate the molecular determinants of sensitivity and resistance of tumor cells towards withanolides. Using $\mathrm{IC}_{50}$ concentrations of eight different withanolides (withaferin $\mathrm{A}$, withaferin $\mathrm{A}$ diacetate, 3-azerininylwithaferin $\mathrm{A}$, withafastuosin $\mathrm{D}$ diacetate, 4-B-hydroxy-withanolide $\mathrm{E}$, isowithanololide $\mathrm{E}$, withafastuosin $\mathrm{E}$, and withaperuvin) and 19 established anticancer drugs, we analyzed the cross-resistance profile of 60 tumor cell lines. The cell lines revealed cross-resistance between the eight withanolides. Consistent cross-resistance between withanolides and nitrosoureas (carmustin, lomustin, and semimustin) was also observed. Then, we performed transcriptomic microarray-based COMPARE and hierarchical cluster analyses of mRNA expression to identify mRNA expression profiles predicting sensitivity or resistance towards withanolides. Genes from diverse functional groups were significantly associated with response of tumor cells to withaferin A diacetate, e.g. genes functioning in DNA damage and repair, stress response, cell growth regulation, extracellular matrix 
components, cell adhesion and cell migration, constituents of the ribosome, cytoskeletal organization and regulation, signal transduction, transcription factors, and others.

Keywords: cancer; drug development; medicinal food; microarray; pharmacogenomics; natural products

\section{Introduction}

Drug resistance and severe adverse side-effects are major obstacles to cancer chemotherapy. Therefore, new therapy options with improved efficacy are urgently required. Nutritional sources such as marine and terrestrial plants are fertile grounds in which to find bioactive constituents with anti-tumor activity. The long-lasting experience of traditional phytotherapy may facilitate the identification of novel treatment strategies. In India, herbs have been used as foods and medicine for millennia. In recent years, the active principles of food and medicinal herbs have been increasingly elucidated, making the active chemical compounds accessible to molecular biological and biochemical research [1-8]. As compounds of Ayurveda (Sankskrit: knowledge of life) may have molecular targets different from those of standard anti-cancer drugs, they are attractive candidates in the search for novel drugs suitable to treat otherwise drug-resistant tumors. These natural compounds may also show reduced side effects on normal organs [9].

Withania somnifera (L.) Dunal (Indian ginseng, winter cherry, Sanskrit: Ashwagandha) belongs to the family of Solanaceae (nightshade plants). In Ayurveda, roots of the plant are either chewed or used to prepare beverages (aqueous decocts). Withania is widely known for its aphrodisiacal, liver tonic, anti-inflammatory, immune-stimulatory, and astringent activities. Furthermore, Withania somnifera is used to treat asthma, ulcers, emaciation, insomnia, senile dementia, tumors, diabetes, neurodegenerative disorders and numerous other symptoms and disorders [10-14].

The major phytochemicals in Withania somnifera and other Withania species are the withanolides, C-28-steroidal lactone triterpenoids built on an intact or rearranged ergostane framework. Within this framework, C-22 and C-26 are appropriately oxidized forming a six-membered lactone ring [15]. Other bioactive constituents in Withania somnifera are alkaloids, such as isopelletierine and anaferine [16].

In addition, Withania somnifera's use in traditional medicine, Withania somnifera extracts as well as isolated withaferin A can exert chemopreventive effects [17-20]. Both substances have been shown to inhibit tumor cell growth in cell lines in vitro, in mouse tumors in vivo, and in human xenograft tumors transplanted into nude mice [21-26].

As is frequently observed with natural products in general, withaferin A exerts its anti-cancer effect by targeting multiple pathways rather than a single target in tumor cells. Withaferin A induces cell cycle arrest in the $\mathrm{G}_{2} \mathrm{M}$ phase [23,24,27-30]. Furthermore, withaferin A inhibits angiogenesis [31,32]. The full range of mechanisms contributing to the anti-cancer activity of withaferin $\mathrm{A}$ is incompletely understood at present. In addition, the cytotoxic and anti-cancer effects of other withanolides have not yet been thoroughly investigated either [33-39]. 
The aims of the present study were, firstly, to investigate whether tumor cells develop cross-resistance between standard anticancer drugs and withanolides and, secondly, to elucidate the molecular determinants of sensitivity and resistance of tumor cells to withanolides. Using $\mathrm{IC}_{50}$ concentrations of withanolides and the most frequently used standard clinical anticancer drugs, we analyzed the cross-resistance profile of 60 cell lines of the National Cancer Institute (NCI), USA [40]. Next, we performed transcriptomic microarray-based COMPARE analyses of mRNA expressions and then subjected the candidate genes to hierarchical cluster analyses to identify mRNA expression profiles, which predict sensitivity and resistance of tumor cell withanolides. Our microarray-based investigation resulted in novel candidate genes associated with the response of cancer cells to withaferin A diacetate. We found that tumor cell response was associated with genes from diverse functional groups (DNA damage and repair, stress response, cell growth regulation, extracellular matrix components, cell adhesion and cell migration, constituents of the ribosome, cytoskeletal organization and regulation, signal transduction, transcription factors, and others), indicating that resistance and sensitivity may be determined by multiple mechanisms.

\section{Results}

\subsection{Cytotoxicity of Withanolides towards Cancer Cells}

Eight withanolides were analyzed. Their chemical structures are shown in Figure 1. These substances were investigated over doses ranging from $10^{-8}$ to $10^{-4} \mathrm{M}$ in 60 cell lines of the NCI, and $\log _{10} \mathrm{IC}_{50}$ values were calculated for each withanolide for each cell line. The mean $\log _{10} \mathrm{IC}_{50}$ values for cell lines of each tumor type are depicted in Figure 2. Withafastuosin D diacetate, withaferin A diacetate, and 4-B-hydroxy-withanolide $\mathrm{E}$ were the most cytotoxic compounds of the panel tested, whereas withafastuosin $\mathrm{E}$ and withaperuvin showed only minimal inhibitory activity towards the cancer cell lines in the panel. Withaferin A, isowithanololide E, and 3-azerininylwithaferin A demonstrated intermediate cytotoxicity (Figure 2). Among a panel of 60 tumor cell lines, leukemia cell lines were on average most sensitive towards withafastuosin $\mathrm{D}$ diacetate, withaferin $\mathrm{A}$ diacetate, 4-B-hydroxy-withanolide E, and withaferin A. Colon cancer lines were on average most sensitive among cell lines derived from solid cancers.

\subsection{Cross-Resistance of the NCI Cell Line Panel Towards Withanolides}

In order to find out whether the cell lines in the NCI panel exhibit cross-resistance to the eight withanolides investigated, we correlated the corresponding $\log _{10} \mathrm{IC}_{50}$ values to each other for each cell line. As shown in Table 1, correlation coefficients of $\mathrm{R}>0.6$ (Pearson's correlation test) were found frequently for correlation among the withanolides, indicating that the cell line panel shows significant cross-resistance towards the various withanolides. Next, the $\log _{10} \mathrm{IC}_{50}$ values of the NCI cell line panel for the eight withanolides were correlated with those for established anticancer agents. Representative drugs from several major cancer drug classes (alkylating agents, platinum compounds, DNA topoisomerase I and II inhibitors, antimetabolites, and mitotic spindle poisons) were chosen. Significant correlations were consistently observed between withanolides and carmustine (BCNU), lomustine (CCNU), and semustine (methyl-CCNU), indicating that many of the NCI cell lines 
demonstrate cross-resistance between withanoloides and these nitrosoureas. The correlations between withanolides and other established anticancer drugs were weak $(\mathrm{R}<0.6)$ (Table 1).

Figure 1. Chemical structures of the 8 withanolides tested.

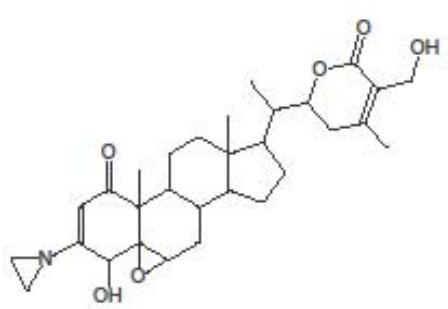

3-azerinidylwithaferin $\mathrm{A}$

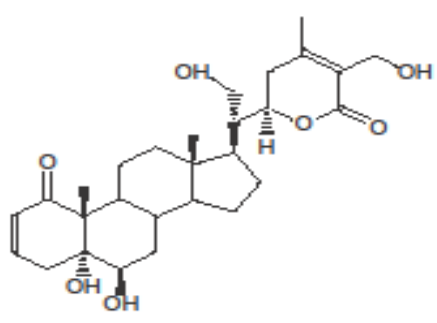

withafastuosin $\mathrm{E}$

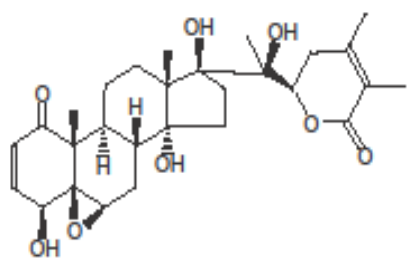

4-B-hydroxy-withanolide E

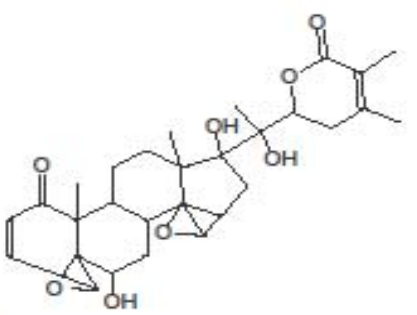

14,15-epoxy-6 a-isowithanolide $E$
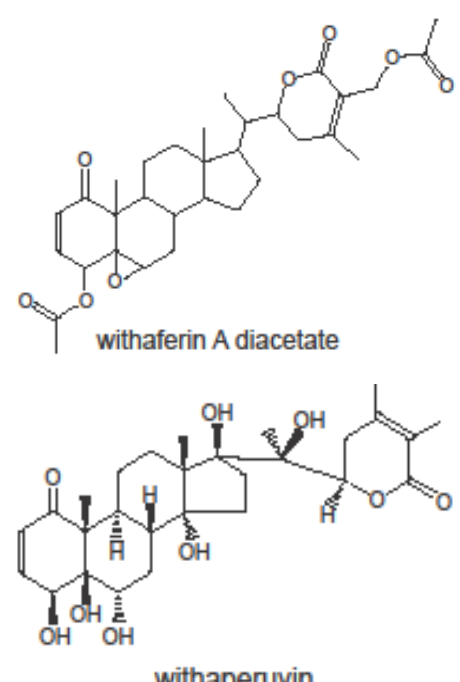

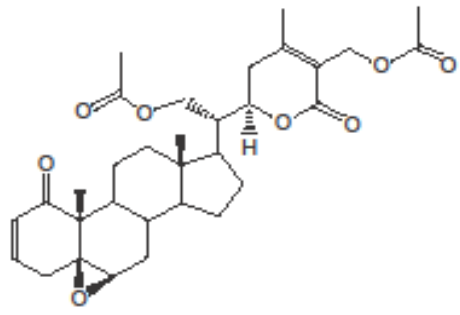

withafastuosin $\mathrm{D}$ diacetate

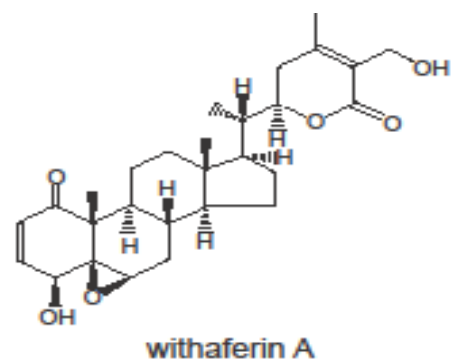

withaferin $\mathrm{A}$

Figure 2. Mean $\log _{10} \mathrm{IC}_{50}$ values of 8 withanolides for tumor cell lines from the NCI drug screening panel as assayed by the sulforhodamine B test.

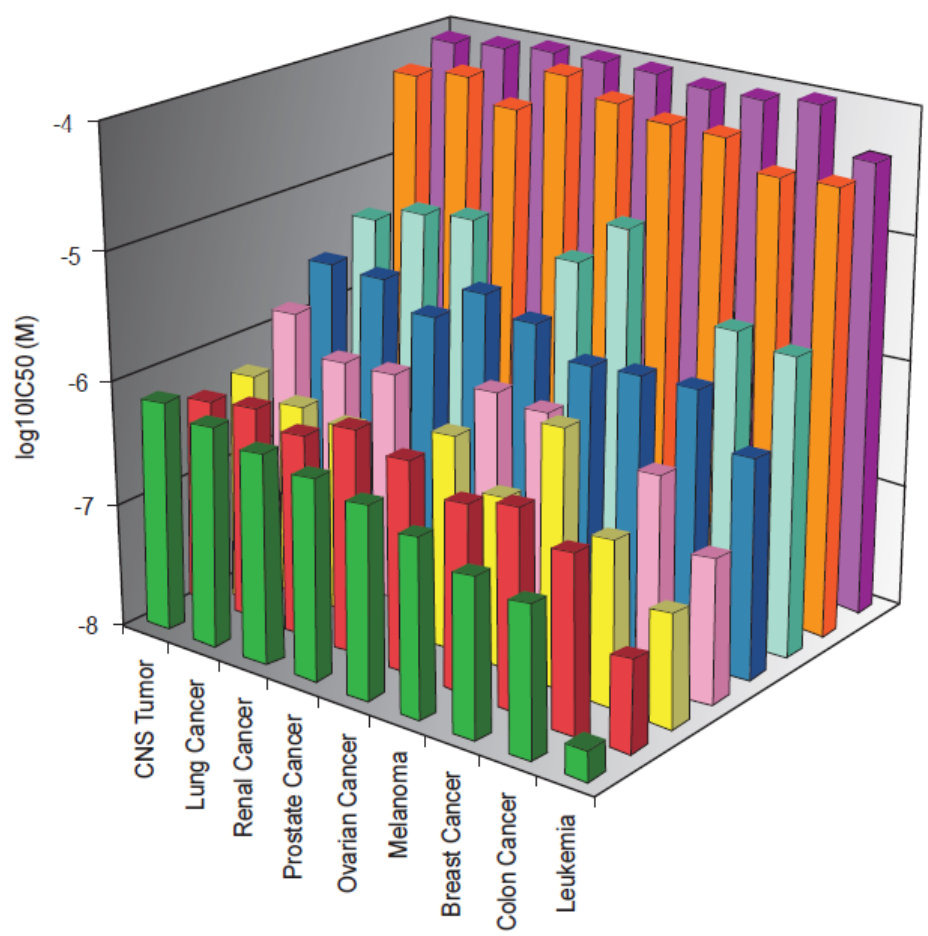

$\square$ Withafuastuosin $\mathrm{D}$ diacetate

$\square$ Withaferin A diacetate

$\square$ 4-B-Hydroxy-withanolide $\mathrm{E}$

$\square$ Withaferin A

$\square$ Isowithanoloide $\mathrm{E}$

$\square$ 3-Azerininylwithaferin A

$\square$ Withafastuosin E

$\square$ Withaperuvin 
Table 1. Cross-resistance of the NCI cell line panel towards withanolides and anticancer drugs.

\begin{tabular}{|c|c|c|c|c|c|c|c|c|c|}
\hline Compounds & $\begin{array}{l}\text { R-/ } \\
\text { P-Values } \\
\end{array}$ & $\begin{array}{l}\text { Withafastuosin } \\
\text { D diacetate }\end{array}$ & $\begin{array}{l}\text { 4-B-hydroxy- } \\
\text { withanolide E }\end{array}$ & $\begin{array}{l}\text { Withaferin } \\
\text { A diacetate } \\
\end{array}$ & $\begin{array}{l}\text { Withaferin } \\
\text { A }\end{array}$ & $\begin{array}{l}\text { Isowithanololide } \\
\text { E }\end{array}$ & $\begin{array}{l}\text { 3-Azerininyl- } \\
\text { withaferin A }\end{array}$ & $\begin{array}{l}\text { Withafastuosin } \\
\text { E }\end{array}$ & Withaperuvin \\
\hline \multicolumn{10}{|l|}{ Withanolides: } \\
\hline \multirow[t]{2}{*}{ Withafastuosin D diacetate } & R-value & & 0.517 & 0.853 & 0.670 & 0.608 & 0.528 & 0.413 & 0.544 \\
\hline & P-value & & $1.00 \times 10^{-4}$ & $9.18 \times 10^{-18}$ & $4.37 \times 10^{-7}$ & $2.08 \times 10^{-7}$ & $8.22 \times 10^{-5}$ & $8.64 \times 10^{-4}$ & $5.11 \times 10^{-6}$ \\
\hline \multirow[t]{2}{*}{ 4-B-hydroxy-withanolide E } & R-value & & & 0.548 & 0.548 & 0.452 & 0.420 & 0.492 & 0.469 \\
\hline & P-value & & & $3.29 \times 10^{-5}$ & $1.05 \times 10^{-4}$ & $7.06 \times 10^{-4}$ & 0.003 & $3.01 \times 10^{-4}$ & $4.48 \times 10^{-4}$ \\
\hline \multirow[t]{2}{*}{ Withaferin A diacetate } & R-value & & & & 0.701 & 0.700 & 0.475 & 0.426 & 0.623 \\
\hline & P-value & & & & $5.95 \times 10^{-8}$ & $3.47 \times 10^{-10}$ & $3.69 \times 10^{-4}$ & $5.98 \times 10^{-4}$ & $6.99 \times 10^{-8}$ \\
\hline \multirow[t]{2}{*}{ Withaferin A } & R-value & & & & & 0.609 & 0.549 & 0.358 & 0.466 \\
\hline & P-value & & & & & $5.70 \times 10^{-6}$ & $8.41 \times 10^{-5}$ & 0.001 & $7.17 \times 10^{-4}$ \\
\hline \multirow[t]{2}{*}{ Isowithanololide E } & R-value & & & & & & 0.348 & 0.439 & 0.513 \\
\hline & P-value & & & & & & 0.008 & $3.94 \times 10^{-4}$ & $1.63 \times 10^{-5}$ \\
\hline \multirow[t]{2}{*}{ 3-Azerininyl-withaferin A } & R-value & & & & & & & 0.280 & 0.349 \\
\hline & P-value & & & & & & & 0.033 & 0.008 \\
\hline \multirow[t]{2}{*}{ Withafastuosin E } & R-value & & & & & & & & 0.291 \\
\hline & P-value & & & & & & & & 0.016 \\
\hline \multicolumn{10}{|l|}{ Alkylating agents: } \\
\hline \multirow[t]{2}{*}{ Carmustine (BCNU) } & R-value & 0.607 & 0.466 & 0.747 & 0.508 & 0.590 & 0.393 & 0.411 & 0.721 \\
\hline & $\mathrm{P}$-value & $1.74 \times 10^{-7}$ & $4.21 \times 10^{-4}$ & $5.64 \times 10^{-12}$ & $2.16 \times 10^{-4}$ & $4.47 \times 10^{-7}$ & 0.003 & $8.27 \times 10^{-4}$ & $5.79 \times 10^{-11}$ \\
\hline \multirow[t]{2}{*}{ Lomustine (CCNU) } & R-value & 0.515 & 0.509 & 0.579 & 0.517 & 0.430 & 0.304 & 0.381 & 0.479 \\
\hline & $\mathrm{P}$-value & $1.51 \times 10^{-5}$ & $1.09 \times 10^{-4}$ & $7.78 \times 10^{-7}$ & $1.65 \times 10^{-4}$ & $3.37 \times 10^{-4}$ & 0.019 & 0.002 & $6.15 \times 10^{-5}$ \\
\hline \multirow[t]{2}{*}{ Semustine (me-CCNU) } & R-value & 0.542 & 0.389 & 0.653 & 0.381 & 0.519 & 0.305 & 0.362 & 0.618 \\
\hline & P-value & $5.63 \times 10^{-6}$ & 0.003 & $1.36 \times 10^{-8}$ & n.s. & $1.48 \times 10^{-5}$ & 0.019 & 0.003 & $1.2 \times 10^{-7}$ \\
\hline \multirow[t]{2}{*}{ Melphalan } & R-value & 0.471 & 0.268 & 0.496 & 0.349 & 0.429 & 0.186 & 0.253 & 0.550 \\
\hline & P-value & $8.47 \times 10^{-5}$ & 0.033 & $3.25 \times 10^{-5}$ & 0.010 & $3.53 \times 10^{-4}$ & n.s. & 0.030 & $3.24 \times 10^{-6}$ \\
\hline \multirow[t]{2}{*}{ Ifosfamide } & R-value & 0.162 & 0.222 & 0.140 & 0.265 & 0.136 & 0.204 & 0.199 & 0.083 \\
\hline & P-value & n.s. & n.s. & n.s. & 0.041 & n.s. & n.s. & n.s. & n.s. \\
\hline \multicolumn{10}{|l|}{ Platin compounds: } \\
\hline \multirow[t]{2}{*}{ Cisplatin } & R-value & 0.199 & 0.110 & 0.296 & 0.191 & 0.282 & 0.050 & 0.131 & 0.379 \\
\hline & P-value & n.s. & n.s. & 0.011 & n.s. & 0.015 & n.s. & n.s. & 0.002 \\
\hline \multirow[t]{2}{*}{ Carboplatin } & R-value & 0.124 & 0.220 & 0.279 & 0.195 & 0.308 & 0.110 & 0.127 & 0.391 \\
\hline & $\mathrm{P}$-value & n.s. & n.s. & 0.016 & n.s. & 0.009 & n.s. & n.s. & 0.001 \\
\hline
\end{tabular}


Table 1. Cont.

\begin{tabular}{|c|c|c|c|c|c|c|c|c|c|}
\hline Compound & $\begin{array}{l}\text { R-/ } \\
\text { P-Value }\end{array}$ & $\begin{array}{l}\text { Withafastuosin } \\
\text { D diacetate }\end{array}$ & $\begin{array}{l}\text { 4-B-hydroxy- } \\
\text { withanolide E }\end{array}$ & $\begin{array}{l}\text { Withaferin } \\
\text { A diacetate }\end{array}$ & $\begin{array}{l}\text { Withaferin } \\
\text { A }\end{array}$ & $\begin{array}{l}\text { Isowithanololide } \\
\text { E }\end{array}$ & $\begin{array}{l}\text { 3-Azerininyl- } \\
\text { withaferin A }\end{array}$ & $\begin{array}{l}\text { Withafastuosin } \\
\text { E }\end{array}$ & Withaperuvin \\
\hline \multicolumn{10}{|c|}{ DNA topoisomerase I inhibitors: } \\
\hline \multirow[t]{2}{*}{ Camptothcin } & R-value & 0.205 & -0.018 & 0.131 & 0.076 & 0.050 & 0.125 & 0.069 & 0.307 \\
\hline & P-value & n.s. & n.s. & n.s. & n.s. & n.s. & n.s. & n.s. & n.s. \\
\hline \multirow[t]{2}{*}{ Topotecan } & R-value & 0.242 & 0.119 & 0.178 & 0.108 & 0.102 & 0.104 & 0.198 & 0.308 \\
\hline & P-value & 0.032 & n.s. & n.s. & n.s. & n.s. & n.s. & n.s. & 0.009 \\
\hline \multicolumn{10}{|c|}{ DNA topoisomerase II inhibitors: } \\
\hline \multirow[t]{2}{*}{ Adriamycin } & R-value & 0.327 & 0.198 & 0.362 & 0.203 & 0.196 & 0.267 & 0.207 & 0.413 \\
\hline & P-value & 0.006 & n.s. & 0.002 & n.s. & n.s. & 0.035 & n.s. & $5.70 \times 10^{-4}$ \\
\hline \multirow[t]{2}{*}{ Daunorubicin } & R-value & 0.440 & 0.213 & 0.447 & 0.305 & 0.210 & 0.333 & 0.247 & 0.460 \\
\hline & P-value & $2.44 \times 10^{-4}$ & n.s. & $1.95 \times 10^{-4}$ & 0.022 & n.s. & 0.011 & 0.033 & $1.25 \times 10^{-4}$ \\
\hline \multirow[t]{2}{*}{ Etoposide } & R-value & 0.237 & 0.063 & 0.244 & 0.256 & 0.199 & 0.150 & 0.109 & 0.246 \\
\hline & P-value & 0.036 & n.s. & 0.031 & 0.047 & n.s. & n.s. & n.s. & 0.030 \\
\hline \multirow[t]{2}{*}{ Teniposide } & R-value & 0.343 & 0.215 & 0.358 & 0.217 & 0.203 & 0.264 & 0.185 & 0.437 \\
\hline & P-value & 0.004 & n.s. & 0.003 & n.s. & n.s. & 0.037 & n.s. & $2.67 \times 10^{-4}$ \\
\hline \multicolumn{10}{|c|}{ Antimetabolites: } \\
\hline \multirow[t]{2}{*}{ 5-Fluorouracil } & R-value & 0.357 & 0.160 & 0.365 & 0.054 & 0.300 & 0.250 & 0.272 & 0.129 \\
\hline & P-value & 0.003 & n.s. & 0.002 & n.s. & 0.011 & 0.046 & 0.021 & n.s. \\
\hline \multirow[t]{2}{*}{ Methotrexate } & R-value & 0.451 & 0.114 & 0.418 & 0.154 & 0.270 & 0.299 & 0.218 & 0.210 \\
\hline & P-value & $1.66 \times 10^{-4}$ & n.s. & $4.94 \times 10^{-4}$ & n.s. & 0.019 & 0.021 & n.s. & n.s. \\
\hline \multicolumn{10}{|c|}{ Mitotic spindle poisons: } \\
\hline \multirow[t]{2}{*}{ Vincristine } & R-value & 0.283 & 0.266 & 0.307 & 0.177 & 0.102 & 0.446 & 0.258 & 0.156 \\
\hline & P-value & 0.015 & 0.034 & 0.009 & n.s. & n.s. & $8.32 \times 10^{-4}$ & 0.028 & n.s. \\
\hline \multirow[t]{2}{*}{ Vinblastine } & R-value & 0.033 & -0.222 & 0.041 & -0.040 & 0.017 & -0.007 & 0.085 & 0.058 \\
\hline & $\mathrm{P}$-value & n.s. & n.s. & n.s. & n.s. & n.s. & n.s. & n.s. & n.s. \\
\hline \multirow[t]{2}{*}{ Paclitaxel } & R-value & 0.283 & 0.193 & 0.308 & 0.143 & 0.048 & 0.399 & 0.304 & 0.315 \\
\hline & P-value & 0.015 & n.s. & 0.009 & n.s. & n.s. & 0.003 & 0.011 & 0.008 \\
\hline \multirow[t]{2}{*}{ Taxotere } & R-value & 0.202 & -0.041 & 0.158 & 0.146 & 0.098 & 0.337 & 0.177 & 0.215 \\
\hline & $\mathrm{P}$-value & n.s. & n.s. & n.s. & n.s. & n.s. & 0.010 & n.s. & n.s. \\
\hline
\end{tabular}

n.s., not significant $(\mathrm{P}>0.05)$. 


\subsection{COMPARE and Cluster Analyses of Microarray-Based mRNA Hybridization:}

COMPARE analyses were performed to obtain a gene expression profile and identify the most up- or down-regulated genes correlated with the $\mathrm{IC}_{50}$ values for the withanolides. We performed COMPARE analyses of $\log _{10} \mathrm{IC}_{50}$ values for the three most cytotoxic withanolides (withafastuosin D diacetate, withaferin A diacetate, and 4-B-hydroxy-withanolide E) and the transcriptomic mRNA-based expression profiles of the NCI cell lines to produce scale indices of correlation coefficients. The microarray data from the NCI website [40] was used to perform further in silico analysis. The mRNA expression levels were determined by microarray analysis [41-43]. We performed a standard COMPARE analysis in which cell lines most inhibited by withanolides (lowest $\log _{10} \mathrm{IC}_{50}$ values) were correlated with the lowest mRNA expression levels of genes. These genes can be considered possible candidate genes in determining cellular resistance to withanolides. Furthermore, reverse COMPARE analysis was carried out, correlating the most inhibited cell lines with the highest gene expression levels. Considering a COMPARE coefficient of $\mathrm{R}>0.6$ as cut-off value, only two genes each fulfilled this criterion in connection with withafastuosin $\mathrm{D}$ diacetate and 4-B-hydroxy-withanolide $\mathrm{E}$. These two compounds were, therefore, excluded from further analyses. The genes whose mRNA expression correlated with withaferin A diacetate are shown in Table 2. Table 2 differs from Table 1 in that it is rearranged in such a way that genes are grouped according to their order in the cluster analysis. This allows one to see which genes were clustered together and which ones were separated. Three main clusters were observed; however, a pattern of genes with similar functions was not seen among the clusters.

Among the genes were genes from diverse functional groups, such as DNA damage response and repair $(R A D 54 L)$, stress response (ANXA2, PPIH, UACA), cell growth regulation (BCAR3, CD53, NASP, TRIM3), extracellular matrix components, cell adhesion and migration (ADAM9, ASAP2, ITGB1, LAMB1), ribosomal proteins (RPS23, RPL5, LOC440055, LOC729362), cytoskeletal organization and regulation (CORO1A, LCP1, PLS3, WAS), signal transduction (ASAP2, BCAR3, DLG2, GNA11, PTPN7, RGS12, RNF138, SH3BP4, TJP1), transcription factors (IFZF1, HCLS1, TRIM3, ZNF112, ZNF228), and others (ALDH7A1, LSM2, MANBAL, NACA, STMN4).

Next, the 40 genes identified by standard and reverse COMPARE analyses were subjected to hierarchical cluster analysis. The dendrogram obtained by this procedure can be divided into three major branches (Figure 3). The distribution of cell lines sensitive or resistant to withaferin A diacetate varies significantly between the branches of the dendrogram. The distribution of cell lines among the dendrogram predicts resistance to withaferin A diacetate with statistical significance $\left(\mathrm{P}=0.00208 \times 10^{-6} ; \chi^{2}\right.$-test; Table 3$)$. 
Table 2. Correlation of constitutive mRNA expression of genes identified by compare analyses with $\mathrm{IC}_{50}$ values for withaferin A diacetate of 60 tumor cell lines.

\begin{tabular}{|c|c|c|c|c|c|}
\hline Symbol & $\begin{array}{l}\text { COMPARE } \\
\text { Coefficient }\end{array}$ & ID No. & Genebank & Name & Function \\
\hline \multicolumn{6}{|l|}{ Cluster 1: } \\
\hline CORO1A & 0.650 & GC9728 & AA047478 & Coronin, actin binding protein, $1 \mathrm{~A}$ & Cytoskeleton component \\
\hline LSM2 & 0.646 & GC31813 & AJ245416 & $\begin{array}{l}\text { LSM2 homolog, U6 small nuclear RNA } \\
\text { associated (S. cerevisiae) }\end{array}$ & Pre-mRNA splicing \\
\hline HCLS1 & 0.676 & GC34797 & X16663 & Hematopoietic cell-specific Lyn substrate 1 & Transcription factor \\
\hline \multicolumn{6}{|l|}{ Cluster 2: } \\
\hline unknown & 0.643 & GC34785 & X79234 & unknown & Unknown \\
\hline PLS3 & -0.606 & GC37799 & M22299 & Plastin 3 & Actin-bundling protein \\
\hline RAD54L & 0.654 & GC32858 & X97795 & RAD54-like (S. cerevisiae) & DNA repair and mitotic recombination \\
\hline RPL5 & 0.648 & GC36655 & U14966 & Ribosomal protein L5 & Structural constituent of ribosome \\
\hline IKZF1 & 0.648 & GC61547 & AI247840 & IKAROS family zinc finger 1 (Ikaros) & Transcriptional regulator \\
\hline DLG2 & -0.612 & GC10718 & R41930 & Discs, large homolog 2 (Drosophila) & $\begin{array}{l}\text { Signal transducer, required for } \\
\text { perception of chronic pain through } \\
\text { NMDA receptor signaling }\end{array}$ \\
\hline RPS23 & 0.669 & GC37806 & D14530 & Ribosomal protein $\mathrm{S} 23$ & Structural constituent of ribosome \\
\hline unknown & 0.674 & GC33814 & D11327 & unknown & Unknown \\
\hline RNF138 & 0.688 & GC67595 & AI608790 & Ring finger protein 138 & ubiquitin-protein ligase, ubiquitinylation \\
\hline unknown & 0.663 & GC31615 & X79234 & unknown & Unknown \\
\hline LCP1 & 0.649 & GC27422 & J02923 & Lymphocyte cytosolic protein 1 (L-plastin) & Actin-binding protein, T-cell activation \\
\hline LAMB1 & -0.610 & GC18026 & AA004918 & Laminin, beta-1 & $\begin{array}{l}\text { Extracellular matrix structural } \\
\text { constituent }\end{array}$ \\
\hline SH3BP4 & -0.608 & GC16071 & W72796 & SH3-domain binding protein & $\begin{array}{l}\text { Signal transducer, functions in } \\
\text { transferrin receptor internalization at the } \\
\text { plasma membrane }\end{array}$ \\
\hline
\end{tabular}


Table 2. Cont.

\begin{tabular}{|c|c|c|c|c|c|}
\hline Symbol & $\begin{array}{l}\text { COMPARE } \\
\text { Coefficient }\end{array}$ & ID No. & Genebank & Name & Function \\
\hline UACA & -0.624 & GC14684 & N66980 & $\begin{array}{l}\text { Uveal autoantigen with coiled-coil domains } \\
\text { and ankyrin repeats }\end{array}$ & Regulation of stress-induced apoptosis \\
\hline BCAR3 & -0.636 & GC14433 & N48319 & Breast cancer anti-estrogen resistance 3 & $\begin{array}{l}\text { Adapter protein for activated growth } \\
\text { factor receptors to signaling pathways } \\
\text { that regulate cell proliferation }\end{array}$ \\
\hline ZNF112 & -0.640 & GC15668 & W15410 & Zinc finger protein 112 homolog (mouse) & DNA binding, transcriptional regulator \\
\hline LOC440055 & 0.721 & GC36107 & AA977163 & Similar to ribosomal protein S12 & Unknown \\
\hline Unknown & -0.601 & GC14769 & N92652 & unknown & Unknown \\
\hline ALDH7A1 & -0.642 & GC16889 & AA024918 & $\begin{array}{l}\text { Aldehyde dehydrogenase } 7 \text { family, member } \\
\text { A1 }\end{array}$ & $\begin{array}{l}\text { Aldehyde dehydrogenase (NAD), } \\
\text { oxidoreductase }\end{array}$ \\
\hline ADAM9 & -0.611 & GC15762 & W47533 & ADAM metallopeptidase domain 9 & $\begin{array}{l}\text { Mediates cell-cell or cell-matrix } \\
\text { interactions }\end{array}$ \\
\hline TRIM3 & -0.616 & GC14991 & N71362 & Tripartite motif-containing 3 & $\begin{array}{l}\text { Transcriptional repressor, control of cell } \\
\text { proliferation }\end{array}$ \\
\hline ITGB1 & -0.638 & GC19072 & AA044261 & $\begin{array}{l}\text { Integrin, beta } 1 \text { (fibronectin receptor, beta } \\
\text { polypeptide, antigen CD29 includes MDF2, } \\
\text { MSK12) }\end{array}$ & integrin binding \\
\hline RGS12 & -0.639 & GC15931 & W67134 & Regulator of G-protein signaling 12 & $\begin{array}{l}\text { Signal transducer, regulator of } \mathrm{G} \\
\text { proteins }\end{array}$ \\
\hline TJP1 & -0.612 & GC12455 & R79560 & Tight junction protein 1 (zona occludens 1 ) & $\begin{array}{l}\text { Signal transduction for tight junction } \\
\text { assembly and stabilizing junctions }\end{array}$ \\
\hline ASAP2 & -0.638 & GC15131 & N70773 & $\begin{array}{l}\text { ArfGAP with } \mathrm{SH} 3 \text { domain, ankyrin repeat } \\
\text { and } \mathrm{PH} \text { domain } 2\end{array}$ & $\begin{array}{l}\text { GTPase activator, Regulates the } \\
\text { formation of post-Golgi vesicles, } \\
\text { modulates cell migration }\end{array}$ \\
\hline STMN4 & -0.650 & GC11515 & H29581 & Stathmin-like 4 & Unknown \\
\hline
\end{tabular}


Table 2. Cont.

\begin{tabular}{|c|c|c|c|c|c|}
\hline Symbol & $\begin{array}{l}\text { COMPARE } \\
\text { Coefficient }\end{array}$ & ID No. & Genebank & Name & Function \\
\hline WAS & 0.654 & GC69113 & AI655719 & $\begin{array}{l}\text { Wiskott-Aldrich syndrome (eczema- } \\
\text { thrombocytopenia) }\end{array}$ & $\begin{array}{l}\text { effector for Rho-type GTPases, regulates } \\
\text { structure and dynamics of the actin } \\
\text { cytoskeleton }\end{array}$ \\
\hline NACA & 0.652 & GC30164 & AF054187 & $\begin{array}{l}\text { Nascent polypeptide-associated complex } \\
\text { alpha subunit }\end{array}$ & $\begin{array}{l}\text { Prevents inappropriate targeting of } \\
\text { non-secretory polypeptides to the } \\
\text { endoplasmic reticulum (ER) }\end{array}$ \\
\hline NASP & 0.657 & GC83792 & AW003362 & $\begin{array}{l}\text { Nuclear autoantigenic sperm protein } \\
\text { (histone-binding) }\end{array}$ & $\begin{array}{l}\text { Involved in DNA replication, normal } \\
\text { cell cycle progression and cell } \\
\text { proliferation }\end{array}$ \\
\hline LOC729362 & 0.679 & GC31589 & T89651 & Similar to ribosomal protein $\mathrm{L} 36 \mathrm{a}$ & Unknown \\
\hline GNA11 & -0.639 & GC31915 & N36926 & $\begin{array}{l}\text { Guanine nucleotide binding protein } \\
\text { (G protein), alpha } 11 \text { (Gq class) }\end{array}$ & $\begin{array}{l}\text { Signal transducer, activator of } \\
\text { phospholipase } \mathrm{C} \text {, GTPase }\end{array}$ \\
\hline unknown & -0.658 & GC32458 & M69013 & unknown & Unknown \\
\hline \multicolumn{6}{|l|}{ Cluster 3: } \\
\hline CD53 & 0.666 & GC89937 & M37033 & cell differentiation antigen $53,32-40 \mathrm{kDa}$ & Growth regulation in hematopoietic cells \\
\hline PPIH & 0.662 & GC28763 & AF016371 & Peptidylprolyl isomerase $\mathrm{H}$ (cyclophilin $\mathrm{H}$ ) & Chaperone \\
\hline PTPN7 & 0.707 & GC90165 & M64322 & $\begin{array}{l}\text { Protein tyrosine phosphatase, non-receptor } \\
\text { type } 7\end{array}$ & $\begin{array}{l}\text { Regulation of } \mathrm{T} \text { and } \mathrm{B}-\text { lymphocyte } \\
\text { development and signal transduction }\end{array}$ \\
\hline ANXA2P3 & -0.652 & GC90123 & M62895 & Annexin A2 pseudogene 3 & Unknown \\
\hline ANXA2 & -0.659 & GC85483 & D00017 & Annexin A2 & $\begin{array}{l}\text { Phospholipase inhibitor, involved in } \\
\text { heat-stress response }\end{array}$ \\
\hline
\end{tabular}

Only genes with correlation coefficients of $>0.6$ or $<-0.6$ were considered. Positive correlation coefficients indicate direct correlations to $\log _{10} \mathrm{IC}_{50}$ values; negative ones indicate inverse correlations. Information on gene functions was taken from the OMIM database, NCI, USA [44] and from the GeneCard database of the Weizman Institute of Science, Rehovot, Israel [45]. 
Figure 3. Dendrogram of hierarchical cluster analysis (complete linkage method) obtained from mRNA expression of 40 genes correlated with $\log _{10} \mathrm{IC}_{50}$ values for withaferin A diacetate. The dendrogram shows the clustering of 60 cell lines according to the mRNA expression profile for genes identified by COMPARE analyses (Table 3).

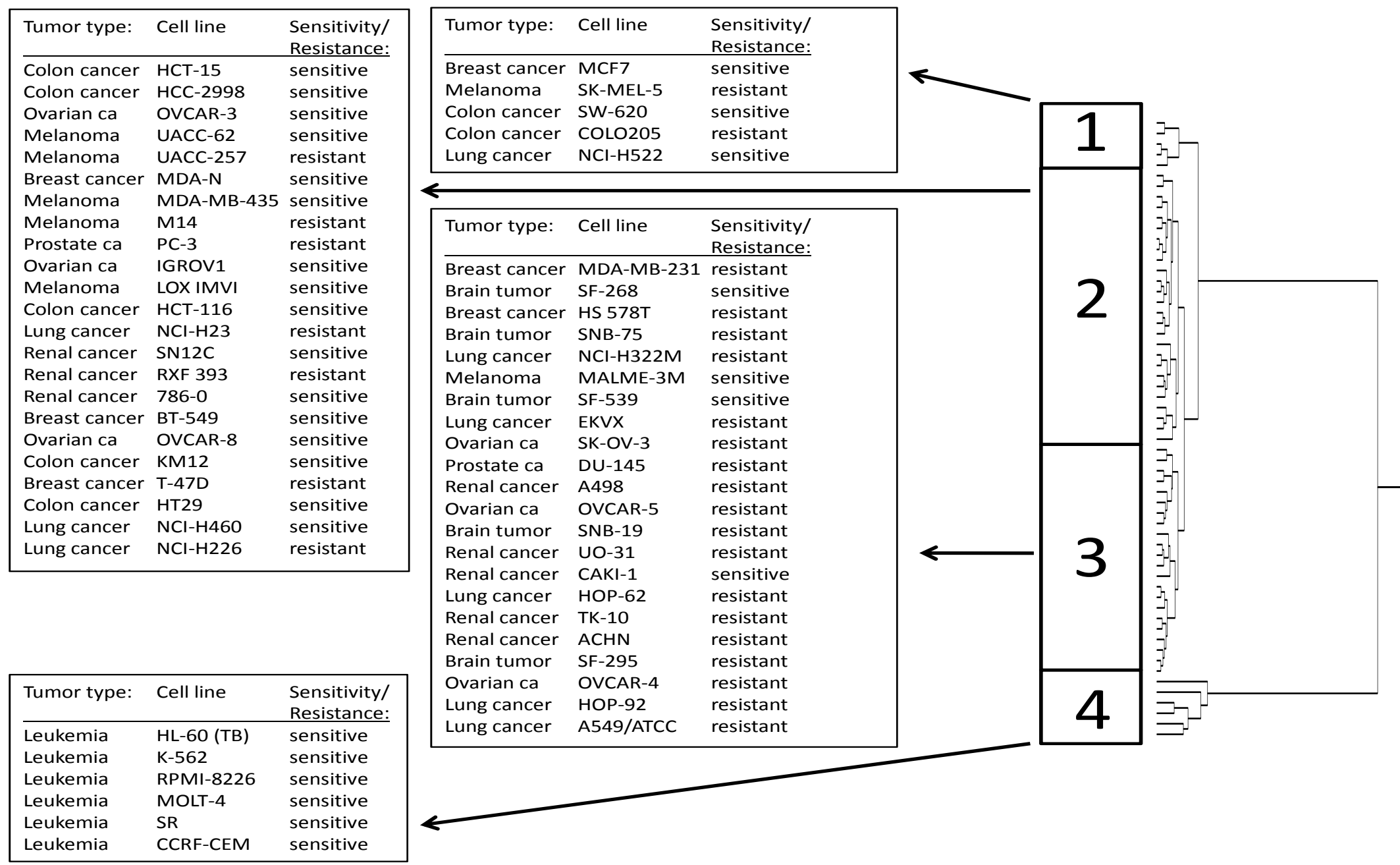


Table 3. Separation of clusters of 60 NCI cell lines obtained by hierarchical cluster analysis shown in Figure 3 in comparison to drug sensitivity. The median $\log _{10} \mathrm{IC}_{50}$ value $(-6.5 \mathrm{~m})$ for each compound was used as cut-off to separate tumor cell lines as being "sensitive" or "resistant".

\begin{tabular}{cccccc}
\hline & Partition & Cluster 1 & Cluster 2 & Cluster 3 & Cluster 4 \\
\hline sensitive & $<-6.5$ & 3 & 9 & 4 & 6 \\
resistant & $>-6.5$ & 2 & 17 & 18 & 0 \\
$\chi^{2}$-test & $\mathrm{P}=0.00208$ & & & & \\
\hline
\end{tabular}

In the present investigation, we showed that six out of the eight withanolides tested exerted profound cytotoxic activity towards tumor cell lines. The cytotoxicity of the withanolides was compared among tumor cell lines belonging to nine different tumor types. We found that leukemic cell lines were particularly sensitive against withanolides. This observation is consistent with previous results obtained by our group that indicate phytochemicals and standard anticancer drugs are frequently more cytotoxic to leukemia cells than to cell lines derived from solid tumors [46-48]. Among the cell lines of solid tumors, colon cancer cells were most sensitive to withaferin A diacetate. A possible explanation might be that the Notch-1 signaling pathway plays an important role in colon carcinogenesis and that withanolides inhibit Notch-1 signaling [49].

Our investigation confirms previous reports showing the cytotoxic activity of withanolides against cancer cells [21,22,24-26,28]. Furthermore, we have analyzed the cross-resistance profile of the cell line panel to different withanolides and between withanolides and established anticancer drugs. By means of Pearson's correlation test, we found that the 60 cell lines exerted significant cross-resistance among withanolides. We observed that cell lines sensitive to one withanolide are likely to be sensitive to other withanolides and vice versa.

We extended this analysis to standard anticancer drugs, and found consistent cross-resistance between withanolides and nitrosoureas. Cross-resistance to other drug classes (platin compounds, DNA topoisomerase I or II inhibitors, antimetabolites, mitotic spindle poisons) was less frequent or not observed. This suggests that withanolides and nitrosoureas may share similar mechanisms of action, e.g., DNA damage of tumor cells. Interestingly, withaferin A has been described as exerting chemo- and radiosensitizing effects on tumors in vitro and in vivo [9,50-52]. It is possible that the interaction of the activity of two treatment substances on DNA may lead to synergistic and sensitizing effects.

As a next step, we correlated the $\mathrm{IC}_{50}$ values of withaferin A diacetate on 60 tumor cell lines with the microarray-based transcriptomic mRNA expression levels of the cell line panel [53] by COMPARE analysis. This approach has been successfully used to unravel the mode of action of novel compounds [53]. Cluster and COMPARE analyses are also useful for comparing gene expression profiles with $\mathrm{IC}_{50}$ values for investigational drugs to identify candidate genes causing drug resistance [54] and to identify prognostic expression profiles in clinical oncology [55].

We identified genes from diverse functional groups that were significantly associated with the response of tumor cells to withaferin A diacetate. These genes were related to DNA damage and repair, stress response, cell growth regulation, extracellular matrix components, cell adhesion and cell 
migration, constituents of the ribosome, cytoskeletal organization and regulation, signal transduction, transcription factors, and others.

The fact that genes associated with sensitivity or resistance against withaferin A diacetate were from diverse functional groups speaks for the multiplicity of mechanisms by which withaferin A diacetate inhibits cancer cells. This so-called "multiplicity of mechanisms" can refer to multiple targets that together lead to multiple effects or it can refer to one target leading to activation or inactivation of multiple downstream pathways. Multi-specificity is a general feature of many natural products; rather than acting on one single target, they affect multiple targets and pathways [8].

The multifaceted nature of withanolides has been previously recognized, e.g., induction of G2M cell cycle arrest [27] and apoptosis [34], inhibition of metastasis [56] and angiogenesis [57], inhibition of the transcription factor NFKB [58] and heat shock protein HSP90 [25,59], and immunomodulation [20]. It is interesting that our transcriptomic approach pointed to additional mechanisms, whose role for response of tumor cells against withanolides have not been considered so far.

For example, RAD54L is involved in recombinatorial DNA repair (via the RAD52 pathway) and dissociates RAD51 from nucleoprotein filaments formed on double-stranded DNA $[45,60]$. The cross-resistance profile of withanolides and nitrosoureas for the 60 cancer cell lines may be explained at least in part by RAD54L. This speculation deserves further investigation in the future.

The significant correlation of the expression of stress response genes to the $\log _{10} \mathrm{IC}_{50}$ values for withanolides indicates that cell lines with high expression of these genes better resist the cytotoxic effects of withaferin A diacetate than cell lines with low expression levels. ANXA2 is a phospholipase inhibitor, which is involved in the heat stress response [45]. Heat shock proteins and chaperones are known to mediate resistance to conventional anticancer drugs [61]. The possibility of ANXA2 playing a role in multidrug resistance and gemcitabine resistance has been suggested [62,63]. PPIH accelerates protein-folding and may act as chaperone, and UACA regulates stress-induced apoptosis by NFkB inhibition [45]. PPIH and UACA have not yet been linked to cellular response to established drugs or withanolides.

Several genes involved in cell growth regulation were significant in our analysis, namely, CD53, BCAR3, TRIM3, and NASP. Although these genes do not belong to the set of classical cell cycle genes, they suggest that withaferin A diacetate may play a role in inhibiting cancer cell proliferation. Many established anticancer drugs also act against cell proliferation. However, most classical anticancer drugs damage not only cancer cells, but normal proliferating cells, which leads to the severe side effects often observed with chemotherapy, e.g., myelosuppression, sterility, gastrointestinal mucosa damage, and alopecia. Whether withaferin A diacetate also exerts detrimental effects on normal tissues is not known and requires further exploration.

Interestingly, a number of genes encoding extracellular matrix (ECM) components and genes involved in cell adhesion and migration correlated with $\log _{10} \mathrm{IC}_{50}$ values for withaferin A diacetate including ADAM9, LAMB1, ITGB1, ASAP2. The ECM, cell adhesion and migration are important components in cancer metastasis and progression. Withanolides are known to inhibit metastasis [56]. We suggest that these four genes identified in our analysis may contribute to the anti-metastatic activity of withaferin A diacetate.

Another interesting finding is that cellular response to withaferin A diacetate was correlated with the expression of genes encoding several constituents of the ribosome. Whereas the role of ribosomal 
proteins in resistance towards established anticancer drugs has not yet been intensively investigated [64], our study indicates that the ribosomal genes RPS23 and RPL5 and the still poorly characterized ribosome-associated genes LOC440055 and LOC729362 affect resistance towards withaferin A diacetate. Ribosomal proteins are often involved in antibiotic resistance. For example, Streptomycin resistance is based on the modification of an aspartic acid moiety in the ribosomal protein S12 [65]. RPS6 is thought to play an important role in controlling cell growth and proliferation by selective translation of particular classes of mRNA [66]. The RPL6 gene plays an important role in the development of drug resistance in leukemia and gastric cancer cells by suppressing drug-induced apoptosis [64,67]. In previous investigations, we observed that genes encoding ribosomal proteins correlated to cellular sensitivity or resistance towards several cytotoxic phytochemicals, including shikonin, resibufogenin, and artesunate $[6,68,69]$.

\section{Experimental Section}

Statistical Analyses: The panel of human tumor cell lines of the Developmental Therapeutics Program of NCI and their testing by sulforhodamine B assay and mRNA microarray hybridization have been described [42,70,71]. The data from these assays can be found at the NCI website [40]. For hierarchical cluster analysis, objects were classified by calculation of distances between individuals $445 \mathrm{rfG}$, by means of the complete linkage method. All objects were assembled into cluster trees (dendrograms). Previously, cluster models were validated for gene expression profiling and for approaching molecular pharmacology of cancer [70,72]. Hierarchical cluster analyses applying the complete linkage method were performed with the WinSTAT program (Kalmia, Cambridge, MA, USA). Missing values were automatically omitted, and the closeness of any two joined objects was calculated by the number of data points they contained. In order to calculate distances between all variables included in the analysis, the program automatically standardizes the variables by transforming the data to a set of values with mean $=0$ and variance $=1$.

For COMPARE analysis, the mRNA expression values of genes of interest and $\log _{10} \mathrm{IC}_{50}$ values for the withanolides were selected from the NCI database [40]. mRNA expression was determined by microarray analyses as reported [70]. COMPARE analysis was performed to produce rank-ordered lists of genes expressed in the NCI cell lines. The methodology has been described previously in detail [73]. Briefly, every gene of the NCI microarray database was ranked for similarity of its mRNA expression to those under the $\log _{10} \mathrm{IC}_{50}$ concentration for the corresponding compound. To derive COMPARE rankings, a scale index of correlation coefficients (R-values) was created. In the standard COMPARE approach, greater mRNA expression in cell lines correlates with enhanced drug resistance, whereas in reverse COMPARE analyses, greater mRNA expression in cell lines indicates drug sensitivity.

Pearson's correlation test was used to calculate significance values and rank correlation coefficients as a relative measure of the linear dependency of two variables. This test was implemented into the WinSTAT Program (Kalmia). The Pearson correlation test was used as a measure for interval-scaled linear correlations. We used the Pearson test rather than the Spearman's Rank correlation test because Spearman's test is based on the equidistance of values, and the values used for our analysis were not equidistant. 
The $\chi^{2}$-test was applied to bivariate frequency distributions of pairs of nominal scaled variables. This test was also implemented into the WinSTAT program (Kalmia Co.). The $\chi^{2}$-test determines the difference between each observed and theoretical frequency for each possible outcome, squares them, divides each by the theoretical frequency, and takes the sum of the results. Performing the $\chi^{2}$-test necessitated defining cell lines as being sensitive or resistant to withaferin A diacetate. This was done by taking the median $\mathrm{IC}_{50}$ value $\log _{10}=-6.5 \mathrm{M}$ for withaferin A diacetate as a cut-off threshold.

\section{Conclusions}

In summary, our microarray-based investigation delivered novel candidate genes that were associated with the response of cancer cells to withaferin A diacetate. These results merit further investigation to prove the causative contribution of these genes to withaferin A diacetate resistance and sensitivity.

\section{References}

1. Koehn, F.E.; Carter, G.T. The evolving role of natural products in drug discovery. Nat. Rev. Drug Discov. 2005, 4, 206-220.

2. Efferth, T.; Li, P.; Kaina, B. From traditional Chinese medicine to rational cancer therapy. Trends Mol. Med. 2007, 13, 353-361.

3. Schmidt, B.M.; Ribnicky, D.M.; Lipsky, P.E.; Raskin, I. Revisiting the ancient concept of botanical therapeutics. Nat. Chem. Biol. 2007, 3, 360-366.

4. Efferth, T.; Kahl, S.; Paulus, K.; Adams, M.; Rauh, R.; Boechzelt, H.; Hao, X.; Kaina, B.; Bauer, R. Phytochemistry and pharmacogenomics of natural products derived from traditional Chinese medicine and Chinese Materia Medica with activity against tumour cells. Mol. Cancer Therap. 2008, 7, 152-161.

5. Li, J.W.H.; Vederas, J.C. Drug discovery and natural products: End of an era or an endless frontier? Science 2009, 325, 161-165.

6. Mahringer, A.; Karamustafa, S.; Klotz, D.; Kahl, S.; Konkimalla, V.B.; Wang, Y.F.; Wang, J.S.; Liu, H.Y.; Boechzelt, H.; Hao, X.J.; Bauer, R.; Fricker, G.; Efferth, T. Inhibition of P-glycoprotein at the blood brain barrier by phytochemicals derived from traditional Chinese medicine. Cancer Genomics Proteomics 2010, 7, 191-205.

7. Efferth, T. Personalized cancer medicine: From molecular diagnostics to targeted therapy with natural products. Planta Med. 2010, 76, 1-12.

8. Efferth, T.; Koch, E. Complex interactions between phytochemicals. The multi-target therapeutic concept of phytotherapy. Curr. Drug Targ. 2011, 12, 122-132.

9. Konkimalla, V.B.; Efferth, T. Molecular mechanisms and interactions responsible for radio- and chemoresistance of tumors and their modulation by natural products from Ayurveda. In Herbal Medicine. A Cancer Chemopreventive and Therapeutic Perspective; Arora, R., Ed.; Jaypee Brothers Medical Publishers Pvt. Ltd.: New Delhi, India; St. Louis, MI, USA, 2010; pp. 513-530.

10. Mishra, L.C.; Singh, B.B.; Dagenais, S. Scientific basis for the therapeutic use of Withania somnifera (ashwagandha): A review. Altern. Med. Rev. 2000, 5, 334-336. 
11. Modak, M.; Dixit, P.; Londhe, J.; Ghaskadbi, S.; Paul, A.; Devasagayam, T. Indian herbs and herbal drugs used for the treatment of diabetes. J. Clin. Biochem. Nutr. 2007, 40, 163-173.

12. Lu, L.; Liu, Y.; Zhu, W.; Shi, J.; Liu, Y.; Ling, W.; Kosten, T.R. Traditional medicine in the treatment of drug addiction. Am. J. Drug Alcohol. Abuse 2009, 35, 1-11.

13. Maurya, R. Chemistry and pharmacology of Withania coagulans: An Ayurvedic remedy. $J$. Pharm. Pharmacol. 2010, 62, 153-160.

14. Ven Murthy, M.R.; Ranjekar, P.K.; Ramassamy, C.; Deshpande, M. Scientific basis for the use of Indian ayurvedic medicinal plants in the treatment of neurodegenerative disorders: Ashwagandha. Cent. Nerv. Syst. Agents Med. Chem. 2010, 10, 238-236.

15. Mirjalili, M.H.; Moyano, E.; Bonfill, M.; Cusido, R.M.; Palazón, J. Steroidal lactones from Withania somnifera, an ancient plant for novel medicine. Molecules 2009, 14, 2373-2393.

16. Rastogi, R.P.; Mehrotra, B.N. Compendium of Indian Medicinal Plants; Central Drug Research Institute: New Delhi, India; St. Louis, MI, USA, 1998; Volume 6.

17. Davis, L.; Kuttan, G. Effect of Withania somnifera on 20-methylcholanthrene induced fibrosarcoma. J. Exp. Clin. Cancer Res. 2000, 19, 165-167.

18. Davis, L.; Kuttan, G. Effect of Withania somnifera on DMBA induced carcinogenesis. J. Ethnopharmacol. 2001, 75, 165-168.

19. Prakash, J.; Gupta, S.K.; Kochupillai, V.; Singh, N.; Gupta, Y.K.; Joshi, S. Chemopreventive activity of Withania somnifera in experimentally induced fibrosarcoma tumours in Swiss albino mice. Phytother. Res. 2001, 15, 240-244.

20. Muralikrishnan, G.; Dinda, A.K.; Shakeel, F. Immunomodulatory effects of Withania somnifera on azoxymethane induced experimental colon cancer in mice. Immunol. Invest. 2010, 39, 688-698.

21. Sharada, A.C.; Solomon, F.E.; Devi, P.U.; Udupa, N.; Srinivasan, K.K. Antitumor and radiosensitizing effects of withaferin A on mouse Ehrlich ascites carcinoma in vivo. Acta Oncol. 1996, 35, 95-100.

22. Srinivasan, S.; Ranga, R.S.; Burikhanov, R.; Han, S.S.; Chendil, D. Par-4-dependent apoptosis by the dietary compound withaferin A in prostate cancer cells. Cancer Res. 2007, 67, 246-243.

23. Stan, S.D.; Zeng, Y.; Singh, S.V. Ayurvedic medicine constituent withaferin a causes G2 and M phase cell cycle arrest in human breast cancer cells. Nutr. Cancer. 2008a, 60, 51-60.

24. Malik, F.; Kumar, A.; Bhushan, S.; Mondhe, D.M.; Pal, H.C.; Sharma, R.; Khajuria, A.; Singh, S.; Singh, G.; Saxena, A.K.; Suri, K.A.; Qazi, G.N.; Singh, J. Immune modulation and apoptosis induction: Two sides of antitumoural activity of a standardised herbal formulation of Withania somnifera. Eur. J. Cancer. 2009, 45, 1494-1509.

25. Yu, Y.; Hamza, A.; Zhang, T.; Gu, M.; Zou, P.; Newman, B.; Li, Y.; Gunatilaka, A.A.; Zhan, C.G.; Sun, D. Withaferin A targets heat shock protein 90 in pancreatic cancer cells. Biochem. Pharmacol. 2010, 79, 542-551.

26. Hahm, E.R.; Lee, J.; Huang, Y.; Singh, S.V. Withaferin a suppresses estrogen receptor- $\alpha$ expression in human breast cancer cells. Mol. Carcinog. 2011, 50, 614-624.

27. Devi, P.U.; Akagi, K.; Ostapenko, V.; Tanaka, Y.; Sugahara, T. Withaferin A: A new radiosensitizer from the Indian medicinal plant Withania somnifera. Int. J. Radiat. Biol. 1996, 69, 193-197. 
28. Stan, S.D.; Hahm, E.R.; Warin, R.; Singh, S.V. Withaferin A causes FOXO3a- and Bim-dependent apoptosis and inhibits growth of human breast cancer cells in vivo. Cancer Res. 2008b, 68, 7661-7669.

29. Mandal, C.; Dutta, A.; Mallick, A.; Chandra, S.; Misra, L.; Sangwan, R.S.; Mandal, C. Withaferin A induces apoptosis by activating p38 mitogen-activated protein kinase signaling cascade in leukemic cells of lymphoid and myeloid origin through mitochondrial death cascade. Apoptosis 2008, 13, 1450-1464.

30. Choi, M.J.; Park, E.J.; Min, K.J.; Park, J.W.; Kwon, T.K. Endoplasmic reticulum stress mediates withaferin A-induced apoptosis in human renal carcinoma cells. Toxicol. In Vitro 2011, 25, 692-698.

31. Mohan, R.; Hammers, H.J.; Bargagna-Mohan, P.; Zhan, X.H.; Herbstritt, C.J.; Ruiz, A.; Zhang, L.; Hanson, A.D.; Conner, B.P.; Rougas, J.; Pribluda, V.S. Withaferin A is a potent inhibitor of angiogenesis. Angiogenesis. 2004, 7, 115-122.

32. Mathur, R.; Gupta, S.K.; Singh, N.; Mathur, S.; Kochupillai, V.; Velpandian, T. Evaluation of the effect of Withania somnifera root extracts on cell cycle and angiogenesis. J. Ethnopharmacol. 2006, 105, 336-341.

33. Choudhary, M.I.; Hussain, S.; Yousuf, S.; Dar, A.; Mudassar, A.-R. Chlorinated and diepoxy withanolides from Withania somnifera and their cytotoxic effects against human lung cancer cell line. Phytochemistry 2010, 71, 2205-2209.

34. Mondal, S.; Mandal, C.; Sangwan, R.; Chandra, S.; Mandal, C. Withanolide D induces apoptosis in leukemia by targeting the activation of neutral sphingomyelinase-ceramide cascade mediated by synergistic activation of c-Jun N-terminal kinase and p38 mitogen-activated protein kinase. Mol. Cancer. 2010, 9, 239.

35. Abdeljebbar, L.H.; Benjouad, A.; Morjani, H.; Merghoub, N.; El Haddar, S.; Humam, M.; Christen, P.; Hostettmann, K.; Bekkouche, K.; Amzazi, S. Antiproliferative effects of withanolides from Withania adpressa. Therapie 2009, 64, 121-127.

36. Xu, Y.M.; Marron, M.T.; Seddon, E.; McLaughlin, S.P.; Ray, D.T.; Whitesell, L.; Gunatilaka, A.A. 2,3-Dihydrowithaferin A-3beta-O-sulfate, a new potential prodrug of withaferin A from aeroponically grown Withania somnifera. Bioorg. Med. Chem. 2009, 17, 2210-2214.

37. He, Q.P.; Ma, L.; Luo, J.Y.; He, F.Y.; Lou, L.G.; Hu, L.H. Cytotoxic withanolides from Physalis angulata L. Chem. Biodivers. 2007, 4, 443-449.

38. Subbaraju, G.V.; Vanisree, M.; Rao, C.V.; Sivaramakrishna, C.; Sridhar, P.; Jayaprakasam, B.; Nair, M.G. Ashwagandhanolide, a bioactive dimeric thiowithanolide isolated from the roots of Withania somnifera. J. Nat. Prod. 2006, 69, 1790-1792.

39. Mathur, S.; Kaur, P.; Sharma, M.; Katyal, A.; Singh, B.; Tiwari, M.; Chandra, R. The treatment of skin carcinoma, induced by UV B radiation, using 1-oxo-5beta, 6beta-epoxy-witha-2-enolide, isolated from the roots of Withania somnifera, in a rat model. Phytomedicine 2004, 11, 452-460.

40. Developmental Therapeutics Program NCI/NIH Hompage. Available online: http://dtp.nci.nih.gov (accessed on 21 May 2012).

41. Alley, M.C.; Scudiero, D.A.; Monks, A.; Hursey, M.L.; Czerwinski, M.J.; Fine, D.L.; Abbott, B.J.; Mayo, J.G.; Shoemaker, R.H.; Boyd, M.R. Feasibility of drug screening with panels of human tumor cell lines using a microculture tetrazolium assay. Cancer Res. 1988, 48, 589-601. 
42. Monk, A.; Scudiero, D.; Skehan, P.; Shoemaker, R.; Paull, K.; Vistica, D.; Hose, C.; Langley, J.; Cronise, P.; Vaigro-Wolff, A. Feasibility of a high-flux anticancer drug screen using a diverse panel of cultured human tumor cell lines. J. Natl. Cancer Inst. 1991, 83, 757-756.

43. Staunton, J.E.; Slonim, D.K.; Coller, H.A.; Tamayo, P.; Angelo, M.J.; Park, J.; Scherf, U.; Lee, J.K.; Reinhold, W.O.; Weinstein, J.N.; Mesirov, J.P.; Lander, E.S.; Golub, T.R. Chemosensitivity prediction by transcriptional profiling. Proc. Natl. Acad. Sci. USA 2001, 98, 10787-10792.

44. OMIM ${ }^{\circledR}$ - Online Mendelian Inheritance in Man $^{\circledR}$ Hompage. Available online: http://www.ncbi.nlm.nih.gov/Omim (accessed on 21 May 2012).

45. GeneCard database. Weizman Institute of Science: Rehovot, Israel, 2012. Available online: http://bioinfo.weizmann.ac.il/cards/index.html (accessed on 21 May 2012).

46. Efferth, T.; Dunstan, H.; Sauerbrey, A.; Miyachi, H.; Chitambar, C.R. The antimalarial artesunate is also a potent anticancer drug. Int. J. Oncol. 2001, 18, 767-773.

47. Efferth, T.; Kahl, S.; Paulus, K.; Adams, M.; Rauh, R.; Boechzelt, H.; Hao, X.; Kaina, B.; Bauer, R. Phytochemistry and pharmacogenomics of natural products derived from traditional Chinese medicine and chinese materia medica with activity against tumor cells. Mol. Cancer Ther. 2008, 7, 152-161.

48. Sertel, S.; Eichhorn, T.; Bauer, J.; Hock, K.; Plinkert, P.K.; Efferth, T. Pharmacogenomic determination of genes associated with sensitivity or resistance of tumor cells to curcumin and curcumin derivatives. J. Nutr. Biochem. 2011, in press.

49. Koduru, S.; Kumar, R.; Srinivasan, S.; Evers, M.B.; Damodaran, C. Notch-1 inhibition by Withaferin-A: A therapeutic target against colon carcinogenesis. Mol. Cancer Ther. 2010, 9, 202-210.

50. Senthilnathan, P.; Padmavathi, R.; Magesh, V.; Sakthisekaran, D. Chemotherapeutic efficacy of paclitaxel in combination with Withania somnifera on benzo(a)pyrene-induced experimental lung cancer. Cancer Sci. 2006, 97, 658-664.

51. Kalthur, G.; Pathirissery, U.D. Enhancement of the response of B16F1 melanoma to fractionated radiotherapy and prolongation of survival by withaferin A and/or hyperthermia. Integr. Cancer Ther. 2010, 9, 370-377.

52. Yang, E.S.; Choi, M.J.; Kim, J.H.; Choi, K.S.; Kwon, T.K. Withaferin A enhances radiation-induced apoptosis in Caki cells through induction of reactive oxygen species, Bcl-2 downregulation and Akt inhibition. Chem. Biol. Interact. 2011, 190, 9-15.

53. Leteurtre, F.; Kohlhagen, G.; Paull, K.D.; Pommier, Y. Topoisomerase II inhibition and cytotoxicity of the anthrapyrazoles DuP 937 and DuP 941 (Losoxantrone) in the National Cancer Institute preclinical antitumor drug discovery screen. J. Natl. Cancer Inst. 1994, 86, 1239-1244.

54. Efferth, T.; Gebhart, E.; Ross, D.D.; Sauerbrey, A. Identification of gene expression profiles predicting tumor cell response to 1-alanosine. Biochem. Pharmacol. 2003, 66, 613-621.

55. Efferth, T.; Olbrich, A.; Bauer, R. mRNA expression profiles for the response of human tumor cell lines to the antimalarial drugs artesunate, arteether, and artemether. Biochem. Pharmacol. 2002, 64, 617-623. 
56. Thaiparambil, J.T.; Bender, L.; Ganesh, T.; Kline, E.; Patel, P.; Liu, Y.; Tighiouart, M.; Vertino, P.M.; Harvey, R.D.; Garcia, A.; Marcus, A.I. Withaferin A inhibits breast cancer invasion and metastasis at sub-cytotoxic doses by inducing vimentin disassembly and serine 56 phosphorylation. Int. J. Cancer. 2011, 129, 2744-2755.

57. Conner, B.P.; Rougas, J.; Pribluda, V.S. Withaferin A is a potent inhibitor of angiogenesis. Angiogenesis 2004, 7, 115-122.

58. Grover, A.; Shandilya, A.; Punetha, A.; Bisaria, V.S.; Sundar, D. Inhibition of the NEMO/IKK $\beta$ association complex formation, a novel mechanism associated with the NF- $\kappa \mathrm{B}$ activation suppression by Withania somnifera's key metabolite withaferin A. BMC Genomics. 2010, 11, 25.

59. Grover, A.; Shandilya, A.; Agrawal, V.; Pratik, P.; Bhasme, D.; Bisaria, V.S.; Sundar, D. Hsp90/Cdc37 chaperone/co-chaperone complex, a novel junction anticancer target elucidated by the mode of action of herbal drug Withaferin A. BMC Bioinformatics 2011, 12, 30.

60. Swagemakers, S.M.; Essers, J.; de Wit, J.; Hoeijmakers, J.H.; Kanaar, R. The human RAD54 recombinational DNA repair protein is a double-stranded DNA-dependent ATPase. J. Biol. Chem. 1998, 273, 28292-28297.

61. Fuqua, S.A.; Oesterreich, S.; Hilsenbeck, S.G.; Von Hoff, D.D.; Eckardt, J.; Osborne, C.K. Heat shock proteins and drug resistance. Breast Cancer Res. Treat. 1994, 32, 67-71.

62. Zhang, F.; Zhang, L.; Zhang, B.; Wei, X.; Yang, Y.; Qi, R.Z.; Ying, G.; Zhang, N.; Niu, R. Anxa2 plays a critical role in enhanced invasiveness of the multidrug resistant human breast cancer cells. J. Proteome Res. 2009, 8, 5041-5047.

63. Gong, X.G.; Lv, Y.F.; Li, X. Q.; Xu, F.G.; Ma, Q.Y. Gemcitabine resistance induced by interaction between alternatively spliced segment of tenascin-C and annexin A2 in pancreatic cancer cells. Biol. Pharm. Bull. 2010, 33, 1261-1267.

64. Chen, H.; Xie, Z.X.; Jiang, H.; Zhang, Z.W.; Wang, G.P. Effect of ribosomal protein L6 on drug resistance and apoptosis in K562/A02 cells. Zhongguo Shi Yan Xue Ye Xue Za Zhi 2007, 15, 292-295.

65. Harris, E.H.; Burkhart, B.D.; Gillham, N.W.; Boynton, J.E. Antibiotic resistance mutations in the chloroplast $16 \mathrm{~S}$ and 23S rRNA genes of Chlamydomonas reinhardtii: Correlation of genetic and physical maps of the chloroplast genome. Genetics. 1989, 123, 281-292.

66. Stolovich, M.; Tang, H.; Hornstein, E.; Levy, G.; Cohen, R.; Bae, S.S.; Birnbaum, M.J.; Meyuhas, O. Transduction of growth or mitogenic signals into translational activation of TOP mRNAs is fully reliant on the phosphatidylinositol 3-kinasemediated pathway but requires neither S6K1 nor rpS6 phosphorylation. Mol. Cell Biol. 2002, 22, 8101-8113.

67. Du, J.; Shi, Y.; Pan, Y.; Jin, X.; Liu, C.; Liu, N.; Han, Q.; Lu, Y.; Qiao, T.; Fan, D. Regulation of multidrug resistance by ribosomal protein 16 in gastric cancer cells. Cancer Biol. Ther. 2005, 4, 242-247.

68. Efferth, T.; Miyachi, H.; Bartsch, H. Pharmacogenomics of a traditional Japanese herbal medicine (Kampo) for cancer therapy. Cancer Genomics Proteomics 2007, 4, 81-92.

69. Sertel, S.; Eichhorn, T.; Sieber, S.; Sauer, A.; Weiss, J.; Plinkert, P.K.; Efferth, T. Factors determining sensitivity or resistance of tumor cell lines towards artesunate. Chem-Biol. Interact. 2010, 185, 42-52. 
70. Scherf, U.; Ross, D.T.; Waltham, M.; Smith, L.H.; Lee, J.K.; Tanabe, L.; Kohn, K.W.; Reinhold, W.C.; Myers, T.G.; Andrews, D.T.; et al. A gene expression database for the molecular pharmacology of cancer. Nat. Genet. 2000, 24, 236-244.

71. Amundson, S.A.; Do, K.T.; Vinikoor, L.C.; Lee, R.A.; Koch-Paiz, C.A.; Ahn, J.; Reimers, M.; Chen, Y.; Scudiero, D.A.; Weinstein, J.N.; et al. Integrating global gene expression and radiation survival parameters across the 60 cell lines of the National Cancer Institute Anticancer Drug Screen. Cancer Res. 2008, 68, 415-424.

72. Efferth, T.; Fabry, U.; Osieka, R. Apoptosis and resistance to daunorubicin in human leukemic cells. Leukemia 1997, 11, 1180-1186.

73. Wosikowski, K.; Schuurhuis, D.; Johnso, K.; Paull, K.D.; Myers, T.G.; Weinstein, J.N.; Bates, S.E. Identification of epidermal growth factor receptor and erbB2 pathway inhibitors by correlation with gene expression patterns. J. Natl. Cancer Inst. 1997, 89, 1505-1515.

(C) 2012 by the authors; licensee MDPI, Basel, Switzerland. This article is an open access article distributed under the terms and conditions of the Creative Commons Attribution license (http://creativecommons.org/licenses/by/3.0/). 